\title{
Journal of the International AIDS

\section{Cost-effectiveness of DRV/r 600/100 mg BID in treatment-experienced, LPV/r-naïve, PI-resistant, HIV-infected adults in the UK, Belgium, Italy and Sweden} K Moeremans ${ }^{1}$, LC Hemmett ${ }^{2}$, J Hjelmgren ${ }^{3}$, G Allegri ${ }^{4}$ and E Smets*5

\author{
Address: ${ }^{1}$ IMS Health HEOR, Brussels, Belgium, ${ }^{2}$ Tibotec, a division of Janssen-Cilag Ltd, Buckinghamshire, UK, ${ }^{3}$ Janssen-Cilag AB, Sollentuna, \\ Sweden, ${ }^{4}$ Janssen-Cilag S.p.A., Cologno Monzese - Milano b, Italy and ${ }^{5}$ Johnson \& Johnson Pharmaceutical Services, Mechelen, Belgium \\ * Corresponding author
}

from Ninth International Congress on Drug Therapy in HIV Infection

Glasgow, UK. 9-13 November 2008

Published: 10 November 2008

Journal of the International AIDS Society 2008, I I (SuppI I):P3II3 doi:I0.II86/I758-2652-II-SI-P3I3

This abstract is available from: http://www.jiasociety.org/content/I I/SI/P3I3

(c) 2008 Moeremans et al; licensee BioMed Central Ltd.

\section{Purpose of the study}

The Phase III TITAN trial (TMC114-C214) evaluated darunavir/ritonavir (DRV/r) 600/100 mg BID vs. lopinavir/ ritonavir (LPV/r) 400/100 mg BID in treatment-experienced, LPV/r-naïve, HIV-infected adults. We determined the cost-effectiveness of DRV/r vs. LPV/r from the perspective of British, Swedish, Italian and Belgian payers in the TITAN trial subgroup with at least one IAS-USA primary protease inhibitor (PI) mutation at baseline. These patients had less advanced HIV disease and a broader degree of prior PI use/failure $(0-\geq 2)$ than those in the DRV Phase IIb POWER trials $(\geq 2)$.

\section{Methods}

An existing Markov model containing six CD4+ T-cell count (CD4 count)-defined health states and a "death" state was adapted to the above mentioned countries. Baseline demographics and CD4 count distribution, antiretroviral drug usage, virologic and immunologic response rates and matching transition probabilities were based on TITAN trial data collected in the modelled subgroup during the first 48 weeks of therapy and from published literature. Patients were assumed to switch to a follow-up combination therapy after failure. For each model state, utility values and mortality rates were obtained from published literature. Costs in each state were obtained from local observational studies and official, local unit costs or from published literature. A lifetime horizon was taken. Discount rates varied according to local guidelines.

\section{Summary of results}

The base-case incremental cost-utility was $€ 18,213$ (£13,111)/QALY, €7,605 (SEK 70,379)/QALY, €17,592/ QALY and $€ 7,990 / Q A L Y$ in the UK, Sweden, Italy and Belgium, respectively. Assuming a threshold of $€ 30,000 /$ QALY, DRV/r remained cost-effective over most parameter ranges tested in extensive one-way sensitivity analyses. Probabilistic sensitivity analysis revealed a probability of $\geq 67 \%$ of an ICER below this threshold in all countries.

\section{Conclusion}

From the British, Swedish, Italian and Belgian payer perspective, DRV/r 600/100 mg BID is predicted to be costeffective vs. LPV/r in the management of LPV/r-naïve, PIresistant, HIV-infected adults with a broad range of prior PI use/failure. 